\title{
Daily Activities Diarist: Supporting Aging in Place with Semantically Enriched Narratives
}

\author{
Georgios Metaxas ${ }^{1}$, Barbaros Metin ${ }^{1}$, Jutta Schneider ${ }^{1}$, Panos Markopoulos ${ }^{1}$, \\ and Boris de Ruyter ${ }^{2}$ \\ ${ }^{1}$ USI Programme, Eindhoven University of Technology, \\ The Netherlands \\ \{g.metaxas, b.metin, j.m.schneider, p.markopoulos\} atue.nl \\ ${ }^{2}$ Philips Research \\ \{boris.de.ruyter@philips.com\}
}

\begin{abstract}
The Daily Activities Diarist is an awareness system that supports social connectedness between seniors living alone and their social intimates. The Daily Activities Diarist extracts automatically an Activity-of-Daily-Life (ADL)-journal from data collected through a wireless sensor network installed at the home of the seniors. We describe the design of the system, its implementation and the lessons from two trials lasting 2 weeks each. The paper makes the case for narrative presentation of awareness information and for seamful design of awareness systems of this ilk.
\end{abstract}

Keywords: Computer-mediated communication, awareness systems, assisted living, ubiquitous computing.

\section{Introduction}

The well known aging of Western societies has prompted a growing interest into technologies that supports "Aging in place" and "Assisted living". Systems designed to support awareness through continual and partly automated flow of information between seniors living alone and their social intimates can help bridge geographical distance, discrepant lifestyles and daily routines, potentially providing peace of mind to both parties and feelings of being connected.

By now, there is a substantial body of work on Awareness systems supporting informal social communication at the home. An early influential project was Casablanca [7] which constituted a first exploration of the relevant design space. It proposed concepts that are still current today such as notice boards shared between households, or using decorative objects (e.g. a lampshade) to provide friends or family with an indication of a user's presence.

The Aroma project [17] let users stay in touch by adding to everyday means of communication (such as telephone and email) with a shared media space. The media space was organized as a pair of windows on different workstations, each displaying abstract visual and auditory effects, all together reflecting the state of affairs at the remote site. The visual effects were represented as an abstract, dynamic painting in 
which the dynamics reflect the changes in the combined auditory and the visual state of the remote site.

Astra [12] was an exploration of intentional communication for the extended family that was shown to enhance feelings of connectedness and to prompt direct communications. Projects such as Intel's CareNet [3] and Honeywell's I.L.S.A [6] have examined the use of similar systems for supporting aging in place; they focus on providing professionals care-givers information about elder's medication, nutrition, falls etc. A related light-weight communication-oriented concept was the DigitalFamily-Portrait (DFP) [19]; DFP was designed to provide peace of mind to adult children regarding a lone parent living at a distance. DFP presents graphically the activity level of the senior and other contextual information at their location (e.g. weather). This system constituted a significant advance over earlier such systems as it was deployed and tested in the field whereas earlier systems (e.g., [15]) were only tested briefly in the lab or activity sensing was simulated by Wizard of Oz techniques (for example, CareNet [3] relied on telephone interviews with participants to feed the display with awareness information). Recent projects such as SharedLife[22] explore the possibilities of extracting and encoding "personal memories" using contextual information and sensor input either for personal use or for sharing among individuals.

Looking at current research prototypes of awareness systems connecting households, it is noteworthy that they are still semantically impoverished with little progress made towards system interpretation of awareness information. The Interliving project [8] explored several communication appliances to connect family members; whereas interesting concepts were produced and the project has accomplished long term field deployment (6 months), the information communicated does not involve any system interpretation. The Digital Family portrait mentioned above [19] only goes so far as visualizing an aggregation of sensor firings over the day rather than attempting a more meaningful interpretation of this data. An important reason for this is that it is difficult to obtain reliable interpretations of user activity and to prevent false alarms. This does not represent only a technical challenge; improvements in technology may improve the quality of the data obtained but the inherent design challenge remains of basing awareness on potentially flawed inferences regarding human activity.

In the present study, we examine the feasibility of providing semantically rich interpretations of sensor activity and applying the concept of 'seamful design' [2], in order to support users who are exposed to the imperfections of the sensing technology. We examine the use of narrative information to disambiguate graphical status presentations of awareness information, in line with Gershon et. al. who argue that images are susceptible to uncertainties and require some declarative statements for clarification [5].

The study reported in this paper explored the feasibility of automatically generating a detailed journal of daily activities, and through several iterations of design and evaluations explored how such information can by usefully presented. This iterative process lead to the lead to the conception and the design of the Daily Activities Diarist, a wireless Activity-of-Daily-Life (ADL)-journal from data collected through a wireless sensor network installed at the home of seniors. Two field trials were conducted with the Daily Acitivities Diarist lasting two weeks each. In each case, the household of an elderly person living alone was connected to that of 
their adult children. The field trials provide an initial assessment of whether awareness of such information is valued by the elderly and their children.

In the remainder of this paper we sketch out the user studies that lead to the design of our awareness system, we explain the motivation behind its design, and summarize its implementation briefly. Finally we describe two trials that we run, for two weeks each and outline future work.

\section{User Studies}

By its nature awareness can be seen as the flip side of privacy [1], requiring the capture and disclosure of information about an individual. Continuous presentation of awareness information about one's social intimates can lead to an information overflow [9] or at least to regular disruption of the receiver of this information. These trade-offs were investigated by a user study that involved interviews, focus group sessions, and questionnaires with both seniors and social intimates.

\section{User Profiles}

In this paper the term 'Senior adults' refers to people over the age of 65 , retired, that have children, and do not suffer from any serious illness. Our target group of senior adults mostly approximates Healthy Hermits [14], i.e. senior individuals remaining in relatively good health yet somewhat withdrawn socially. Healthy hermits have experienced at least one life-changing event such as the death of a spouse. They don't like their isolation or that they're expected to act like old people. Adults who are 85 years old constitute the upper age limit of this population, as they tend to become frailer and have more health problems after that age. The senior adults targeted live alone. However, they have a good and close relationship with their children. They communicate with each other on a regular basis.

The second target group consists of 'intimate socials' (or social intimates) of the senior adults, such as sons and/or daughters (Neustaedter et al. [16]). This group consists of people in the age of 45 to 60 years old who have a close personal relationship with their parents, but live a certain distance away from them (e.g. at a different city), and mostly have a different life rhythm than their parents.

\section{Interviews}

Interviews were conducted with seniors ( $\mathrm{N}=4,69$ to 85 years old) and intimate socials $(\mathrm{N}=3,54-57)$ to realize their attitudes and patterns of communication. Staying up-todate with events in the other's life, communicating their own experiences, exchanging practical information, showing interest, reinforcing the relationship and giving or receiving emotional support were reported as the main reasons of communication by both groups. Elderly are more interested in everyday happenings in the lives of their social intimates; the latter want to know more about general activities of the elderly in the day and if there have any needs and/or problems.

The interviews revealed that seniors fear to bother or to annoy their children when they contact them too often or too long. On the other hand they feel checked up on by daily phone calls from their children. They don't want to share bad moods and feelings with their social intimates. 
The intimate socials reported wanting to know how their parents feel, i.e. what their moods are. It is also important for them to know where they are (in or out the house), and if they are asleep or not. All in all they would like to get a general impression of their daily activities.

In both groups it was apparent that women more often mediate social and emotional contacts. Elderly men are more likely to initiate contact only if they have a clear and practical purpose in mind, such as a question they want to ask. The abovementioned findings are consistent with earlier studies, such as [11] and [13], the NESTOR-LSN survey [4] and the Digital Family Portraits project [15] .

\section{Focus groups}

To confront the target groups with the notion of Awareness Systems and to evaluate our initial concept designs two focus group sessions were held; one with senior adults $(\mathrm{N}=6,75-86)$ at the 'Wilgenhof' elderly home and one with intimate socials $(\mathrm{N}=5,45$ 54) at the Philips High Tech Campus.

More specifically a collection of 9 related design concepts were presented to both groups, and used as a discussion basis in the focus group sessions (figure 1 shows two of the mockups we used as a prompt to examine a scenario). The mockups were presented as paper prototypes through a mechanical frame, that allowed flipping between the various drawings. This helped simulate the dynamic behavior of the system, showing the transaction between different awareness information at different moments in the day. It is worth mentioning that all the examples used in focus group sessions, were based on an analogy with a real world window. For example, figure 1, displays a greeting scenario where a social intimate is seeing through the window her parent greeting when the day starts.

Both groups were quite positive about prospective system-attributes such as its unobtrusiveness. Interestingly the main concerns about privacy arose at the socialintimate side, who did not wish to compromise the privacy of their elderly relative, while a typical response given by elderly was "Anyhow, we know everything about each other". Social-intimates expressed an interest in being aware of the physicalstatus on the other side (e.g., sleeping, eating etc.), critical events such as rapid decline of the parent's health. They were also interested in knowing the feelings and moods of their parents; however seniors were reluctant regarding the communication of negative feelings.
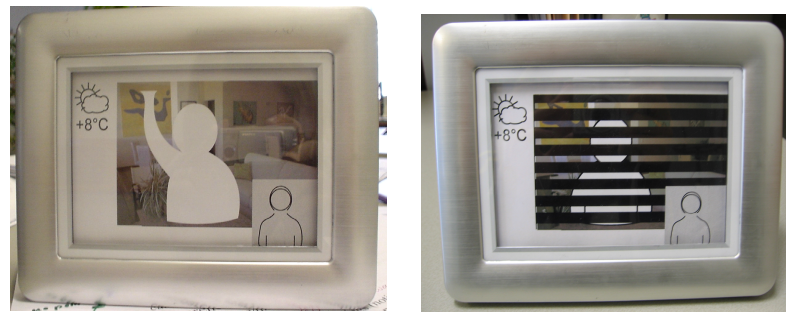

Fig. 1. Two of the prompts used in the focus group sessions 


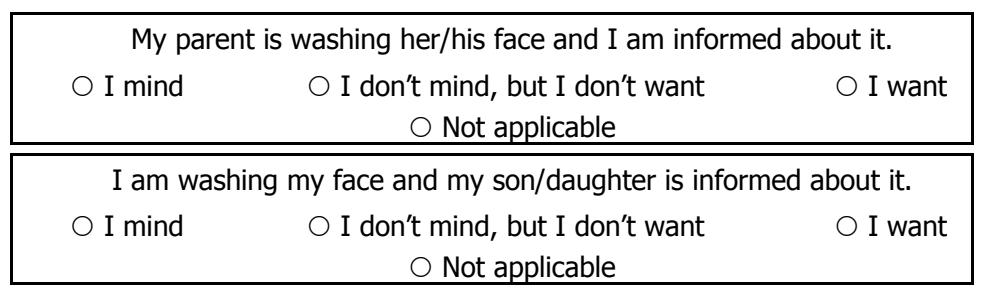

Fig. 2. Example question from the questionnaire for the elderly (top), and for the social intimates (bottom)

\section{Questionnaires}

Rather than taking a technology centric perspective of in surveying the acceptance of communicating different kinds of information that are possible to sense automatically, we aimed to understand privacy issues and user preferences regarding awareness information without reference to the way this information can be captured. A questionnaire was assembled based on various inventories of activities-of-daily-life (ADL) to examine what kind of activities the seniors $(\mathrm{N}=10$, avg. age 81) want (or don't mind) to share on one hand, and what their social-intimates ( $N=15$, avg. age 45) want to be informed about on the other.

The questionnaire was compiled from different published inventories such as the "Activities of daily life list" [11], the "Instrumental activities of daily life" [10] and the "Advanced activities of daily life" [18]. These inventories provide comprehensive lists of activities at minute-level detail initially intended for profiling the level of selfefficacy of an individual. By asking subjects to indicate the degree to which they would like their social intimates to be aware of the activities listed, we get a comprehensive understanding of their need to share awareness information. The activities that were more of interest for our design were:

- Day-to-day maintenance activities such as feeding, sleeping, personal hygiene, dressing, etc.

- Instrumental activities, such as shopping, calling, cooking, doing the laundry, using the phone, etc.

- Daily life concern activities, which arise out of individual abilities and interests, such as social activities.

The format of these questions is illustrated in figure 2. Each questionnaire included 49 such items. The outcome dictated that home presence/absence, bed-occupation, visiting friends/having visitors, followed by other activities like having a walk, cooking, shopping etc. could be shared from the senior side to the social-intimate side without jeopardizing their privacy. Another interesting finding was that the senior adults overall wanted to share good moods and feelings $(\mathrm{N} \geq 9)$ but not negative ones $(\mathrm{N} \leq 3)$.

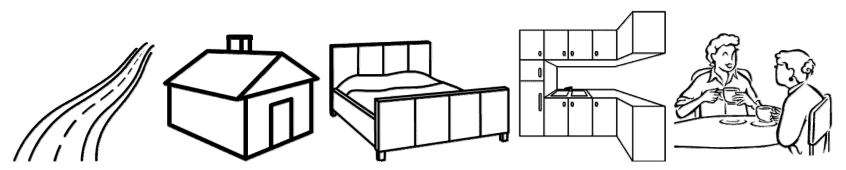

Fig. 3. Graphical presentations of "away", "at home", "in bed", "at the kitchen", and "with Visitors" 


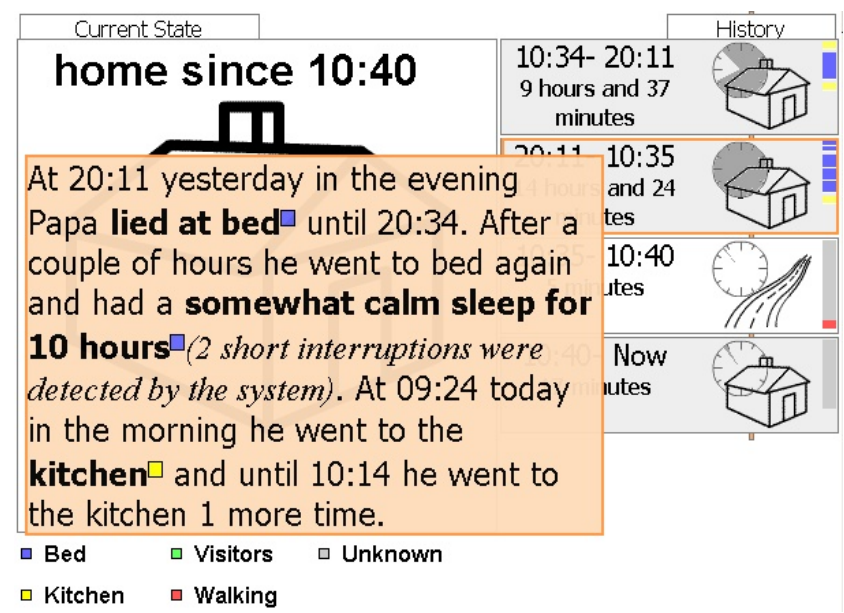

Fig. 4. Screen shot of subject's A1 Daily-Activity-Diarist, showing all three levels of detail presented to users

\section{Prototype Design and Implementation}

\section{Conceptual design}

Based on the user studies described and more specifically on the senior adults' activities that could be shared with their intimate socials without putting privacy at risk, we chose activities such as walking, sleeping, having visitors, cooking and eating, to populate an auto-generated ADL-journal. Due to the stated preference of elderly to keep negative feelings private, we discarded from this journal moods and feelings. Apart from the synchronous exchange of real time activity data, the conceptual design was supplied with a history of logged activity data, to bridge the different life paces of the user groups.

Further to the graphical display of activities, we decided also to use a narrative presentation with more detailed feedback and reasoning about the displayed activities. The narrative feedback was chosen to address the problems that may rise from falsealarms and user-misinterpretations, when graphical information visualization is invoked. Our goal was to minimize these problems by providing semantic-cues and explanatory-statements using narration as a complement to graphical visualization of the extracted activities.

In order to maintain peripheral-awareness and light-weight interaction with the end-users (i.e. the social intimates), the features of the ADL-journal were presented through an 'interactive dynamic poster', assembling the following goals:

- Major changes in the poster can be identified from a long distance using icons (see fig. 3); therefore social intimates can maintain a peripheral awareness of the elderly activities at the other side. 
- Distance is an element of interaction; the closer the user gets to the poster/display, the more detailed information she can get. More detailed information is offered as a historical list on the right column of the display (see fig 4)

- When the poster/display is within reach-of-hands, the user can directly invoke a detailed narrative explaining the system status and activity journal created (fig 4). In this way social intimates can acquire more information about the system's reasoning regarding the displayed activities.

\section{Architectural overview}

In figure 5 we see an overview of the system architecture. The Sensor-Network at the elderly side collects raw data that are pulled from the ADL-State Extractor. The ADLState Extractor abstracts in software terms the sensors and interprets the collected signals to predefined ADL states. These states are time-stamped and pushed to the ADL-State Database Host where they are stored in a database for later process. When it is needed (e.g., on request, or on specific intervals), the ADL-Semantics Extractor pulls the corresponding states from the database, filters and transforms them to an ADL journal that is described in a XML-semantics file. Depending on the configuration, the XML data are pushed to, or pulled by the Presentation Server, which does the final transformation to HTML code. The location of Presentation Server is resolved from a Point to Point Server that redirects the Client requests to the resolved URL.

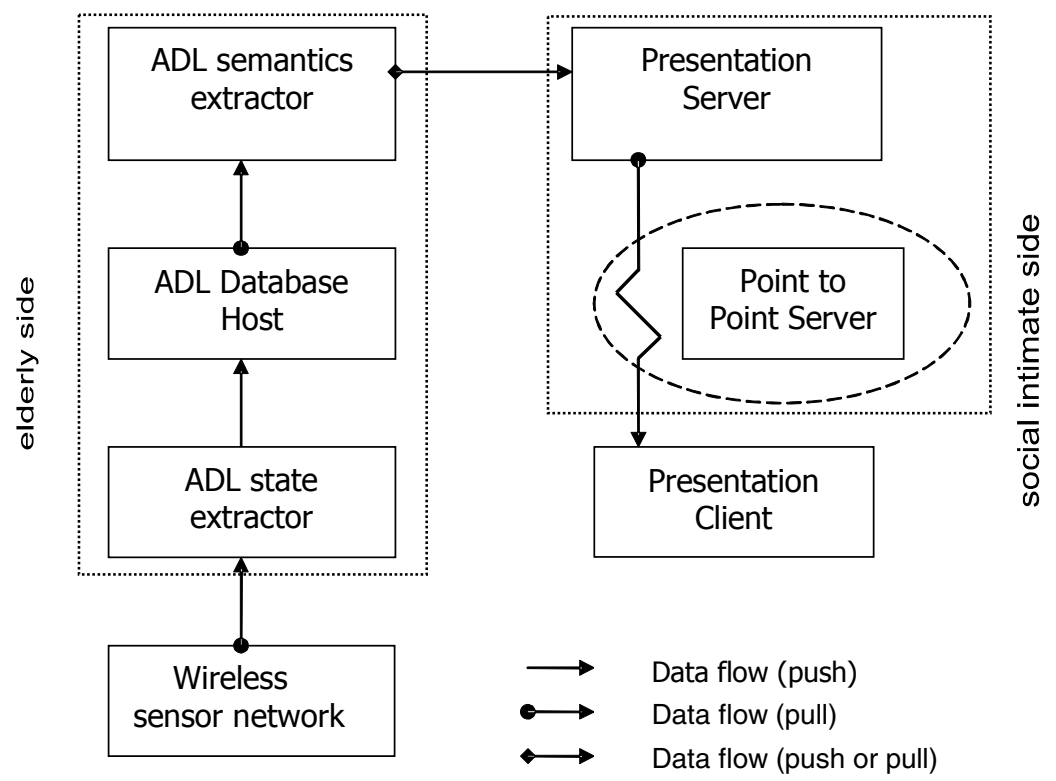

Fig. 5. Overview of the prototype architecture, its components, and their location in the current experimental configuration 


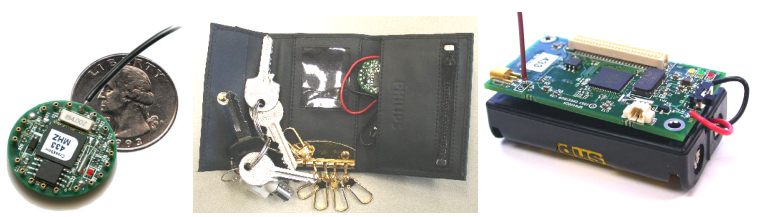

Fig. 6. From left to right: mica2dot mote, key fold with mica2dot, and mica2 mote

\section{Wireless Sensor Network and ADL-State-Extraction}

A wireless sensor network was used to collect data from the elderly homes. Presence at home, mobility, sleep, and other activities are extracted using the raw data collected from this first layer. The wireless sensors are abstracted in software terms by ADLState-Extractors, which transform the collected raw data to ADL-states.

The Sensor network is responsible for all the collected data that are fed in to the system. Presence at home, activity, sleep, and other activities should be extracted by using the data collected from this first layer. The implementation of this layer was based on Crossbow wireless sensors. These are small programmable wirelesscommunication motes that can be connected to a variety of sensors. The Crossbow motes are equipped with TinyOS, an open-source operating system designed for wireless embedded sensor networks.

With the aforementioned framework the Crossbow motes were programmed to support some basic functionality that initiates the activity extraction at the ADL-State extractor side.

\section{Presence and Mobility detection}

A subject's presence or absence detection is done using the Crossbow MICA2dot wireless coin-size mote (Figure 6). The mote is placed in the subject's key-fold. When the subject is present at home, any signal from the sensor can be detected from the sensor and interpreted as presence, and vice versa when a subject is absent.

In order to detect the mobility of a subject, an accelerometer sensor is added to the mote. When the subject is away the accelerometer records the subject's activity. The activity data can be interpreted later by the system, when the subject is back at home.

In order to make possible the above and to maintain low power consumption, the MICA2dot is programmed to transmit its state every 10 seconds. Additionally, every 30 seconds it gets 1 second of high sampling acceleration data. When the energy of the one-second acceleration samples is higher than a predefined 'walking' threshold, the 30 seconds interval is marked as high activity and vice versa.

On the ADL-state extraction side, the sensor is abstracted in software terms, and the collected data are transformed to presence/absence states. The high and low activity counters are compared with the latest known values in order to calculate the subject's mobility while the subject was away. Furthermore, some filters were introduced to overcome problems with lost messages. For example, although the system expects a signal from the sensor every 10 seconds, the subject is considered to be away only if no signal is received for more than a minute. 


\section{Kitchen activity detection}

Activity in the kitchen and other rooms was identified by monitoring the light condition of cupboards. For example, by monitoring light emittance inside a refrigerator we can tell when the door was opened. The combination of these events from various sources, like cupboards and refrigerators, is used to extract information regarding activity in the kitchen (and possibly other rooms).

For the above purpose we equipped MICA2 motes (Figure 7 left) with photosensors and programmed the motes to measure light emittance every 2 seconds. If the measured light exceeds a 'cupboard open' threshold, the sensor sends a signal to the system. To avoid battery drain, the sensors are programmed not to transmit more than once per minute.

On the ADL-state-extraction side, a collection of these motes is abstracted in software, and the collected data are transformed to kitchen activity data. A sequence of 'open cupboards' is interpreted as high kitchen activity with the corresponding duration and intensity.
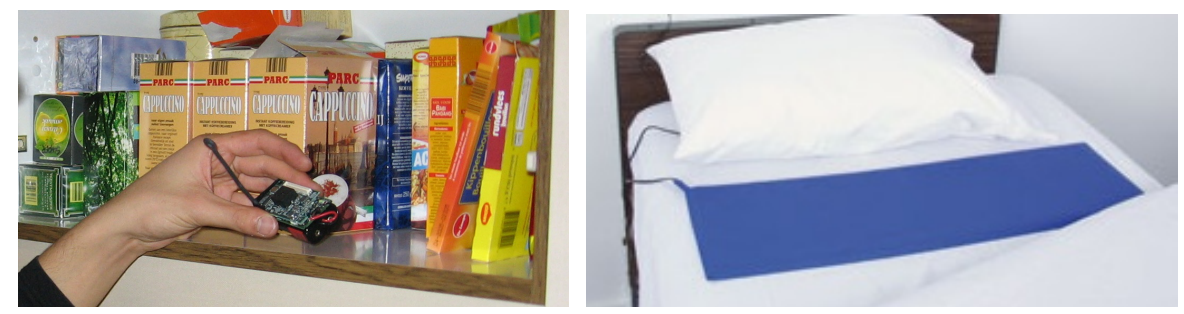

Fig. 7. Placing a wireless mote with a photo sensor in a cupboard(left), an illustration of a pressure pad used for bed-activity detection (right)

\section{Bed/Chair/Visitors activity detection}

A combination of a MICA2 mote and a pressure-pad (Figure 7 right) is used to detect whether a subject is lying on a bed or sitting on a chair. The MICA2 is programmed to make a measurement of the pressure-pad's analog output every 10 seconds, and the measured value is then transmitted to the system.

On start-up, the ADL-state-extraction component makes the assumption that initially the subject is not lying. Therefore the first readings are used to calculate a 'lying' threshold. The same assumption is done when the subject is away, making possible to adapt the 'lying' threshold to the changing physical condition of the pressure-pad. A sensor-reading higher than the 'lying' threshold is translated as a 'bed-active' state and vice versa. Time delay filters are used to overcome lost signals from the MICA2 mote similar to the presence detection.

Furthermore, on the ADL-state-extraction side, a collection of these motes can be used to detect the presence of visitors (e.g. when more than one chair's state is active, the system turns on the 'visitors' state).

\section{ADL-Semantics Extractor and State Database Host}

The states that are extracted from the ADL-state-extraction components are stored in a database at the ADL-State Database Host as a sequence of states. This sequence however may contain logical errors, or reliability errors that cannot be addressed from 
the previous layer (ADL-state-extraction). For example, a pressure-pad could turn on before presence is detected due to network traffic, or the presence sensor may not be detected at some intervals due to poor network signal resulting in a series of falsely alternating present/absent states.

The ADL-semantics extractor is a software component that is aimed at resolving these issues, by further processing the extracted state-data, and transforms the later to a nested XML ADL-journal. The XML formatted journal allows flexibility on the final rendering, and enables a higher level of semantic analysis.

\section{Presentation Server}

The presentation server makes the final analysis and rendering of the XML semantics to HTML. The tree structure of the XML ADL-journal is transformed using XSL to an HTML document that presents the argumentation regarding the presented activities in order to avoid misunderstandings and to give access to more detailed information. For example, when the duration of a "bed" state is less than three hours the interpretation is "nap", or when a "bed" state is interrupted more than 3 times the interpretation is "disrupted sleep" and so on.

The HTML document contains explanations like: "At 23:30 yesterday in the evening John went to bed and had a somewhat disrupted sleep until 07:45 today in the morning. Then (at 08:20) he went to the kitchen for half an hour". Further more, the system explains its argumentation on user request (e.g. "somewhat disrupted sleep" expands to " 2 interruptions were detected by the system at 1:45 and at 4:30....". In figures 4 and 8 one can notice an actual instance from a subject's ADL-journal with a narrative explanation of some extracted activities.

\section{Evaluation}

Two field trials were conducted each involving two households, that of a senior participant and that of their social intimates. Each trial lasted two weeks. Our aim was to explore the overall experience of living with such a system, patterns of usage to the extent allowed by the duration of the study and affective benefits and costs incurred.

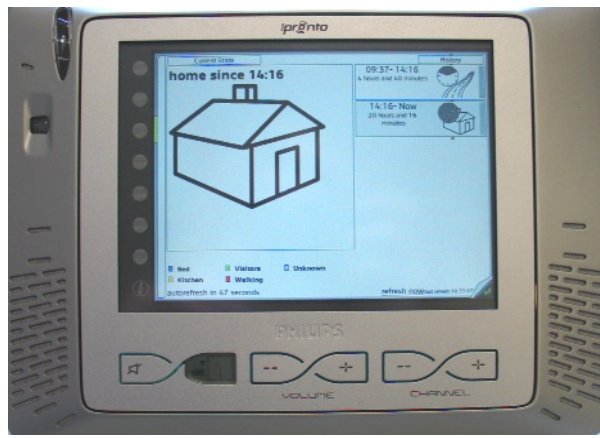

Fig. 8. Daily Activities Diarist displayed on a Philips iPronto device, showing the current state (left) and the history (right) 


\section{Participants}

Family A included a male senior (80) (subject A1), his son (44) (subject A2), daughter in law (43) (subject A3) and their two children. The senior A1 is in good health but uses a so-called 'walker' when going out. Therefore, trips on his-own are restricted to, e.g., getting the newspaper or visits to neighbors. However, he is still an active person.

Family B included a female senior (85) (subject B1), her daughter (57) (B2) and her son in law (57) (B3). The senior B1 is in good health and although she also uses a 'walker' to go out, she is still very mobile.

\section{Apparatus and Maintenance}

The system was installed in the home of the senior participants. Both apartments were similar in size and layout (small bedroom, living room, kitchen, hallway, and bathroom). In their children's homes two Philips iPronto devices (originally intended as 'smart remote controls) were used to display the ADL journals.

In the end of the first week batteries were changed as scheduled, and then the system was left to run for seven more days. Interviews with each participant were conducted at the end of the second week. We also used standardized questionnaires to measure social connectedness using the ABC questionnaire [21] and well being through the subjective complaints questionnaire [20]. Given the small sample size these were not intended for quantitative analysis, but to prepare larger scale trial and to see if any interesting variations in connectedness/well being could be indicated by our participants.

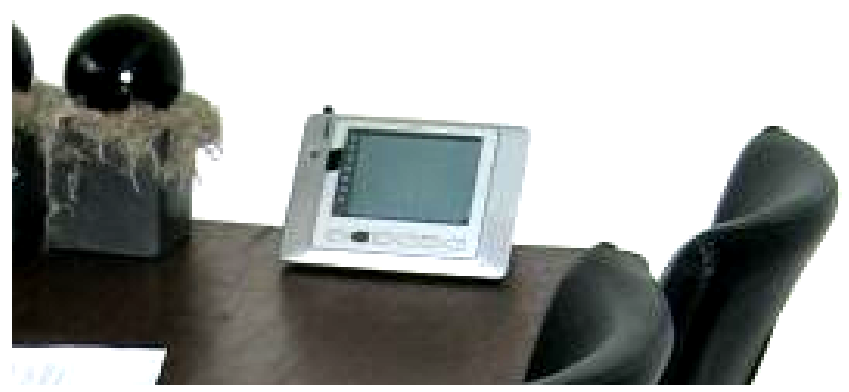

Fig. 9. Presentation Client (iPronto) at participant's B2/B3 living room

\section{Results}

Social intimates at both trials found the given information in the story telling function sufficiently explanatory, although they didn't all use it equally often - "The story telling function was very explanatory, the information which I needed was all provided" - subject A2. Subjects A2, and A3 consulted the narrative regularly (e.g., after waking up and after work). The other subjects were not that interested in acquiring in depth information so frequently.

Participants, A2 and A3, had to get accustomed to the system the first day, but as early as the second day they started relying upon it. Participant A2 used the system to check whether his father was at home or not before calling him, sometimes he 
checked what he had been doing during the day, if he was sleeping, etc. Some of the information they were interested in was not supported by the system's configuration "I would like to know if my father is within 'de Akkers', or outside 'de Akkers' or if he is at another place, such as the supermarket" - subject A2. However, the information provided was experienced overall by participant A2 and A3 as sufficient and appreciated as meaningful.

In contrast, during the second trial technical problems lead to different reactions by participants B2, B3 who found no value in the information provided: "I did not really trust the system, it malfunctioned a lot, and I had to reset it quite a few times." subject B2. Also, they did not feel comfortable with the unidirectional nature of the system fearing that it compromised their parents' privacy - "My mother (in law) should be able to make contact through the system when she wants."- Subject B3, "The system makes me feel as a spy, and it makes her feel as being spied" - subject B2. An erroneous system operation caused subject B2 to be unnecessarily alarmed regarding her mother; also the constant flow of information on her mother's activities made her nervous. She did not however react by immediately calling or visiting her "Yesterday the system showed that my mother was out at night, and this kept us awake all night. However, we were hesitant to disturb her." - subject B2. Both participants thought the system should be made more obtrusive for alerting to critical or life-threatening events.

The participants noted that they prefer to be informed if sensors or other system parts not obvious to them malfunction, e.g., when batteries drain, or when the network is down. This could prevent them from misinterpretations caused by system failures.

From their side, seniors expressed no complaints about the sensors installed in their homes, claiming that they were oblivious to them. Perhaps surprisingly, none of the seniors felt their privacy was invaded. "No privacy issues... it is my son"- subject A1. This however should not be assumed as sufficient evidence that no privacy issues arise; a longer term trial would be more likely to uncover situations where privacy could be compromised or for both parties to better appreciate the privacy risks involved.

An unexpected positive finding (provisional given our small sample) was the reduction of agoraphobia of both participating seniors as measured by post-trial questionnaires. Both seniors reported going out more often than usual; this may be attributed to the safety they felt knowing that their children are aware of their activities.

\section{Conclusion}

Journaling ADLs and displaying them as peripheral social awareness cues can potentially help elderly and their social intimates to be connected. We have argued that a narrative presentation of awareness information that provides rich semantic feedback regarding the system reasoning offers practical advantages over impressionistic graphical presentations of the instantaneous status of the elderly. Clearly a larger empirical basis is needed, but it has to be noted that the difference between these displays is more reliably appreciated by test-participants during actual rather use of the system in the field, rather than lab-based evaluations. 
Running longer term field trials of such systems poses significant challenges, as is evident from current state of the art. Longer term field trials of communication systems rely on capture and communication of raw audio-video data or text/graphical input by users. For example, the Interliving project involved field trials of up to $\mathrm{x}$ months of their communication appliances. On the other hand, the technical and social challenges of installing sensor based awareness systems at the homes of participants result in a long set-up phase to get the system reliable and a comparatively shorter term deployment and actual use of the system. For example, Mynatt et al report actual use of their DFP system by one subject for one week after a set up period of about a year.

Clearly, sensor based awareness systems need to become more robust and easily configurable, so that deployment is faster at many different households. In order to enable longer term user trials with larger sets of participants, we are currently reengineering the Daily Activities Diarist to support end-user configurability of the information flow between the connected households. This is necessary to adapt to the different social and physical contexts of the participants' homes and to allow them to provide personalized descriptions of locations and activities that are meaningful to them and their social intimates.

Awareness systems even when aiming for peace of mind have a safety critical nature; the affective costs of an occasional malfunction can outweigh their benefits as they can unsettle relatives mistakenly. The need to portray the seams of the system, as argued recently by Chalmers et al [2], can be an appropriate design approach in order to avoid false alarms and to provide more meaningful explanations. For example, an other step towards seamful design would be to use network-health and battery-status metrics when reporting activities in order to insert into the narrative confidence qualifications, e.g. "your parent is probably outside", or "Papa might had a disturbed sleep" where "probably" and "might" can be expanded at the request of the user to an explanation about the sensor-network health and battery-status on demand.

\section{References}

1. Boyle, M., Greenberg, S.: The Language of Privacy: Learning from Video Media Space Analysis and Design. ACM ToCHI 12(2), 328-370 (2005)

2. Chalmers, M., Galani, A.: Seamful Interweaving: Heterogeneity in the Theory and Design of Interactive Systems. In: Proc. DIS 2004, pp. 243-252. ACM, New York (2004)

3. Consolvo, S., Roessler, P., Shelton, B.E.: The CareNet Display: Lessons learned from an in-home evaluation of an ambient display. In: Davies, N., Mynatt, E.D., Siio, I. (eds.) UbiComp 2004. LNCS, vol. 3205, pp. 22-29. Springer, Heidelberg (2004)

4. Dykstra, P.A., Knipscheer, P.M.: The availability and intergenerational structure of family relationships. In: Knipscheer, C.P.M., de Jong Gierveld, J., Tilburg, T.G., Dykstra, P.A. (eds.) Living arrangements and social networks of older adults, pp. 37-58 (1995)

5. Gershon, N., Page, W.: What storytelling can do for information visualization. Communications of the ACM 44(8), 31-37 (2001)

6. Haigh, H.Z., Kiff, L.M., Myers, J., Guralnik, V., Kirschbaum, K., Phelps, J., Plocher, T., Toms, D.: The Independent LifeStyle Assistant ${ }^{\mathrm{TM}}$ (I.L.S.A.): Lessons Learned. In: Proc. AAAI, pp. 852-857 (2004) 
7. Hindus, D., Mainwaring, S.D., Leduc, N., Hagström, A.E., Bayley, O.: Casablanca: Designing social communication devices for the home. In: Proceedings CHI 2001, pp. 325-332. ACM, New York (2001)

8. Hutchinson, H., Mackay, W., Westerlund, B., Bederson, B.B., Druin, A., Plaisant, C., Beaudoin-Lafon, M., Conversy, S., Evans, H., Hansen, H., Roussel, N., Eiderbäck, B., Lindquist, S., Sundblad, Y.: Technology Probes: Inspiring Design for and with Families. Proceedings CHI 2003, CHI Letters, 5 (1), pp. 17-24 (2003)

9. Khan, J.V., Markopoulos, P., Mota, S.A., IJsselsteijn, W.A., de Ruyter, B.: Intra-family communication needs; how can Awareness Systems provide support? In: 2nd International Conference on Intelligent Environments (IE06), pp. 84-89 (2006)

10. Lawton, M.P., Brody, E.M.: Assessment of older people: self-maintaining and instrumental activities of daily living. Gerontologist 9, 179-186 (1969)

11. Mahoney, F.I., Barthel, D.W.: Functional evaluation: the Barthel Index. Maryland State Medical Journal 14, 61-66 (1965)

12. Markopoulos, P., Romero, N., Baren, J.v., IJsselsteijn, W., de Ruyter, B., Farshchian, B.: Keeping in Touch with the Family: Home and Away with the ASTRA Awareness System. In: Proc. CHI 2004, ACM, New York (2004)

13. Melenhorst, A.S., Rogers, W.A., Caylor, E.: The use of communication technologies by older adults: exploring the benefits from the users' perspective. In: Proceedings HFES (2001)

14. Moschis, G.P.: Life Stages of the Mature Market. American Demographics, 44-50 (1996)

15. Mynatt, E.D., Rowan, J., Jacobs, A., Craighill, S.: Digital Family Portraits: Supporting Peace of Mind for extended Family Members. In: Proceedings CHI 2001, pp. 333-340 (2001)

16. Neustaedter, C., Elliot, K., Greenberg, S.: Understanding Interpersonal Awareness in the Home. In: ACM CHI 2005 Workshop on Awareness systems (2005)

17. Pedersen, E.R., Sokoler, T.: AROMA: Abstract Representation of Presence Supporting Mutual Awareness. In: Proceedings of ACM SIGHI Conference on Human Factors in Computing systems, pp. 234-241 (1997)

18. Reuben, D.B, Laliberte, L., Hiris, J., Mor, V.: A hierarchical exercise scale to measure at the Advanced Activities of Daily Living (AADL) level. Journal of the American Geriatric Society 38, 855-861 (1990)

19. Rowan, J., Mynatt, E.D.: Digital family portrait field trial: Support for aging in place. In: Proc. CHI 2005, pp. 521-530. ACM, New York (2005)

20. SCL-90-R: Symptom Checklist-90-R: Administration, Scoring \& Procedures Manual LR Derogatis - 1994 - National Computer Systems, Inc

21. van Baren, J., IJsselsteijn, W.A., Romero, N., Markopoulos, P., de Ruyter, B.: Affective Benefits in Communication: The development and field-testing of a new questionnaire measure. In: PRESENCE 2003, Aalborg, Denmark (October 2003)

22. Wahlster, W., Kroner, A., Heckmann, D.: SharedLife: Towards Selective Sharing of Augmented Personal Memories. In: Stock, O., Schaerf, M. (eds.) Reasoning, Action and Interaction in AI Theories and Systems. LNCS (LNAI), vol. 4155, pp. 327-342. Springer, Heidelberg (2006) 